\title{
Necessary and sufficient conditions of freezing phenomena of quantum discord under phase damping
}

\author{
Bo You and Li-Xiang Cen* \\ Department of Physics, Sichuan University, Chengdu 610064, China.
}

(Dated: May 11, 2018)

\begin{abstract}
We investigate the freezing phenomenon of quantum discord occurring in phase damping noise processes. By relating the expression of the time variation of the discord to the convex function of relative entropy, we obtain the necessary and sufficient conditions of the phenomenon for standard Bell-diagonal states. These conditions are applicable also to the phenomenon occurring in a nonMarkovian dephasing process. Moreover, we show that the same condition and phenomenon coincide in a new sort of Bell-diagonal states beyond the standard form.

PACS numbers: 03.67.Mn, 03.65.Yz, 05.70.Fh
\end{abstract}

Quantum discord is regarded as a significant characteristic of quantumness [1, 2] and has attracted much attention in recent literatures [3]. Different from entanglement, quantum discord could capture nonclassical correlations arising in separable states, which has been found to exist in a quantum algorithm without entanglement [4, 5]. Besides the fundamental theoretic interest upon quantum mechanics itself, an appeal of the study on this subject thereby lies in the possibility to process quantum information in a noisy environment since pure entangled states are rather fragile in the real world. Recent studies on the dynamics of quantum discord in various noisy environment have manifested many fascinating features of it [6-13]. It was shown that discord is more robust than entanglement for both Markovian and nonMarkovian dissipative processes [ 6 , 7]. Moreover, the discord of a sort of Bell-diagonal states undergoing a phase damping process was shown to exhibit a freezing phenomenon [11 13], i.e., the amount of discord would be unaffected for a finite period with the system suffering subsequently a sudden change from classical to quantum decoherence.

A bipartite state $\rho_{A B}$ is said to have nonzero quantum discord if $\rho_{A B}$ can not be written as a classicalquantum form, i.e., $\rho_{A B} \neq \sum_{i} p_{i}\left|i_{A}\right\rangle\left\langle i_{A}\right| \otimes \rho_{i}^{B}$. Based on this benchmark, several measures have been proposed to quantify the amount of quantum discord. An information-theoretic measure of it [1, 2] is defined as $Q_{A B} \equiv I\left(\rho_{A B}\right)-I_{c}\left(\rho_{A B}\right)$, where $I\left(\rho_{A B}\right)=S\left(\rho_{A}\right)+$ $S\left(\rho_{B}\right)-S\left(\rho_{A B}\right)$ represents the total correlations of $\rho_{A B}$ and $S(\rho)=-\operatorname{Tr}\left(\rho \log _{2} \rho\right)$ specifies the von Neumann entropy; $I_{c}\left(\rho_{A B}\right)=\max _{\Pi_{A}} I\left[\Pi_{A}\left(\rho_{A B}\right)\right]$ represents the classical correlations of $\rho_{A B}$ and the maximum in it is taken over all projective measurements $\Pi_{A}$ on the subsystem $A$. Other quantifiers of quantum discord include the geometric measure based on the square norm of trace distance [14], and distance-based measures either in terms of the relative entropy [15] or in terms of the fidelity [16].

Generally, the dynamics of the above measures of dis-

*Electronic address: lixiangcen@scu.edu.cn cord under decoherence will manifest distinct behaviors from each other quantitatively or even qualitatively, which suggests the complexity of the issue of decoherence from different perspectives. For the species of Belldiagonal states, it is applaudable that the amount of discord defined via the information-theoretic measure is identical to that defined via the distance measure in terms of the relative entropy [15]. At this stage, the freezing and sudden-transition phenomena of discord exhibited in the Bell-diagonal states are of particular interest as the dynamical behaviors of both quantum and classical correlations there are consistent from different views related to the two kind of measures. We mention that comparing with those sudden-transition phenomena without exhibiting plateaus [ $8-10]$, the freezing dynamics of discord requires more critical conditions. Up to our knowledge, this freezing phenomenon was demonstrated mostly in systems with initial states that are of standard Bell-diagonal form with rank two [11 13, 17, 18]. A complete description of the condition related to the phenomenon is still lacking.

In this paper we investigate necessary and sufficient conditions of the freezing phenomenon of quantum discord occurring in the phase damping process. By elaborating the time variation of the discord of standard Belldiagonal states, we associate the expression of it with the function of relative entropy. This enables us to give a complete description of conditions for the phenomenon by virtue of the convexity property of the relative entropy. The overall geometric structure is then depicted in the parametric space for the collection of states that satisfy these conditions. It is shown that the derived conditions are applicable also to the phenomenon occurring in the non-Markovian dephasing process. Finally, we display that the same condition and phenomenon coincide in a new sort of Bell-diagonal states beyond the standard form.

Time variation of quantum discord of Bell-diagonal states under phase damping. The phasing damping dynamics we are going to investigate characterizes the type of quantum noise process that induces loss of quantum coherence but without energy exchange. For a two-level system, the dynamical evolution of the state under the 
phase damping channel is described as

$$
\rho(t)=\left(\begin{array}{ll}
\rho_{11}(0) & d(t) \rho_{12}(0) \\
d(t) \rho_{21}(0) & \rho_{22}(0)
\end{array}\right)
$$

where $d(t)$ depicts the degradation of the coherence and it often takes an exponential form for a Markovian dephasing process. Hereafter, we shall introduce a notation $q \equiv 1-d(t)$ and treat it a parametrized time.

Let us consider the local phase damping process on a two-qubit system that initially inhabits in the standard Bell-diagonal state

$$
\rho_{A B}=\frac{1}{4}\left(I_{4}+\sum_{i=1}^{3} c_{i} \sigma_{i}^{A} \otimes \sigma_{i}^{B}\right)=\sum_{i=1}^{4} \lambda_{i}\left|\psi_{i}\right\rangle\left\langle\psi_{i}\right|
$$

where $\sigma_{i}$ 's are Pauli operators and $\left|\psi_{i}\right\rangle$ 's are standard Bell bases of the form $\left|\psi_{1,3}\right\rangle=\frac{1}{\sqrt{2}}(|00\rangle \pm|11\rangle),\left|\psi_{2,4}\right\rangle=$ $\frac{1}{\sqrt{2}}(|01\rangle \pm|10\rangle)$. The corresponding eigenvalues $\lambda_{i} \geq 0$ $(i=1, \cdots, 4)$ relate to the parameters $c_{i}$ 's via

$$
\lambda_{1,3}=\frac{1}{4}\left[1 \pm c_{1} \mp c_{2}+c_{3}\right], \quad \lambda_{2,4}=\frac{1}{4}\left[1 \pm c_{1} \pm c_{2}-c_{3}\right] .
$$

As one of the subsystems $A$ or $B$ passes through the channel of Eq. (1), the evolving state $\rho_{A B}(q)$ will remain to be of form of Eq. (2) and the corresponding eigenvalues

$$
\begin{aligned}
& \lambda_{1,3}(q)=\lambda_{1,3} \pm \frac{q}{2}\left[\lambda_{3}-\lambda_{1}\right] \\
& \lambda_{2,4}(q)=\lambda_{2,4} \pm \frac{q}{2}\left[\lambda_{4}-\lambda_{2}\right]
\end{aligned}
$$

The amount of discord defined via the two kind of measures, the information-theoretic measure and the distance measure of relative entropy, coincides for this state and it can be derived analytically as [19]

$$
Q_{A B}(q)=1+\sum_{i=1}^{4} \lambda_{i}(q) \log _{2} \lambda_{i}(q)+h_{2}\left(x_{M}\right)
$$

Here, $h_{2}(x)$ accounts for a binary entropy function $h_{2}(x)=-x \log _{2} x-(1-x) \log _{2}(1-x)$, and $x_{M}(q)=$ $\left[1+c_{M}(q)\right] / 2$ in which $c_{M}(q) \equiv \max \left\{\left|c_{1}(q)\right|,\left|c_{2}(q)\right|,\left|c_{3}\right|\right\}$ and $c_{1,2}(q)=(1-q) c_{1,2}$.

The variation of the discord depends on its local analytical property, recorded as $Q_{A B}^{(i)}(q)(i=1,2,3)$ for the corresponding time intervals in which $c_{M}(q)$ equals to $\left|c_{1}(q)\right|,\left|c_{2}(q)\right|$, or $\left|c_{3}\right|$, hence $x_{M}(q)=\lambda_{1}(q)+\lambda_{2}(q)$, $\lambda_{2}(q)+\lambda_{3}(q)$, or $\lambda_{1}+\lambda_{3}$, respectively [20]. Consider first the situation of $c_{M}(q)=\left|c_{3}\right|$. In this case the term $h_{2}\left(x_{M}\right)$ in Eq. (5) becomes a constant, therefore one obtains

$$
\frac{\mathrm{d}}{\mathrm{d} q} Q_{A B}^{(3)}(q)=-\frac{\mathrm{d}}{\mathrm{d} q} S_{A B}(q)
$$

It is direct to observe that for a Markovian channel, the above variation is always negative since the entropy of
$\rho_{A B}(q)$ should be monotonically increasing under such a dephasing channel. In order to derive further the variation of $Q_{A B}^{(1,2)}(q)$, we introduce the notation of relative entropy: $H_{n}(x \| y)=\sum_{i=1}^{n} x_{i}\left(\log _{2} x_{i} / y_{i}\right)$, where $\left\{x_{i}\right\}$ and $\left\{y_{i}\right\}$ stand for two $n$-component random variables. By virtue of the following relation

$$
\begin{aligned}
& \frac{\mathrm{d}}{\mathrm{d} q} \lambda_{1,3}(q)= \pm \frac{1}{2(1-q)}\left[\lambda_{3}(q)-\lambda_{1}(q)\right] \\
& \frac{\mathrm{d}}{\mathrm{d} q} \lambda_{2,4}(q)= \pm \frac{1}{2(1-q)}\left[\lambda_{4}(q)-\lambda_{2}(q)\right]
\end{aligned}
$$

we are able to work out that

$$
\frac{\mathrm{d}}{\mathrm{d} q} Q_{A B}^{(1)}(q)=\frac{1}{2(1-q)}\left[H_{2}(\mu \| \nu)-H_{4}(\xi \| \eta)\right],
$$

where $\mu(\nu)$ and $\xi(\eta)$ account for probability distributions specified as

$$
\begin{aligned}
& \mu(q)=\left\{x_{M}, 1-x_{M}\right\}, \quad \nu(q)=\left\{1-x_{M}, x_{M}\right\} \\
& \xi(q)=\left\{\lambda_{1}(q), \lambda_{2}(q), \lambda_{3}(q), \lambda_{4}(q)\right\} \\
& \eta(q)=\left\{\lambda_{3}(q), \lambda_{4}(q), \lambda_{1}(q), \lambda_{2}(q)\right\} .
\end{aligned}
$$

A similar expression can be obtained for $\mathrm{d} Q_{A B}^{(2)}(q) / \mathrm{d} q$ merely through exchanging the roles of $\lambda_{1}(q)$ and $\lambda_{3}(q)$ in Eq. (8).

The explicit expression of the local variation of discord derived above enables us to analyze its dynamical behavior conveniently. It is readily seen, from the joint convexity property of relative entropy [21], that the terms in Eq. (8) satisfy

$$
H_{2}(\mu \| \nu) \leq \alpha H_{2}\left(\xi_{1} \| \eta_{1}\right)+\beta H_{2}\left(\xi_{2} \| \eta_{2}\right)=H_{4}(\xi \| \eta),
$$

where $\alpha=\lambda_{1}(q)+\lambda_{3}(q), \beta=\lambda_{2}(q)+\lambda_{4}(q)$, and $\xi_{i}$ and $\eta_{i}$ are variables of probability distributions given by

$$
\begin{aligned}
& \xi_{1}(q)=\frac{1}{\alpha}\left\{\lambda_{1}(q), \lambda_{3}(q)\right\}, \eta_{1}(q)=\frac{1}{\alpha}\left\{\lambda_{3}(q), \lambda_{1}(q)\right\} \\
& \xi_{2}(q)=\frac{1}{\beta}\left\{\lambda_{2}(q), \lambda_{4}(q)\right\}, \eta_{2}(q)=\frac{1}{\beta}\left\{\lambda_{4}(q), \lambda_{2}(q)\right\} .
\end{aligned}
$$

Thus one derives $\mathrm{d} Q_{A B}(q) / \mathrm{d} q \leq 0$ in all time intervals for the Markovian process. We note that this monotonicity of quantum discord is a natural consequence since the Markovian dephasing channel is always unital [16].

Conditions for the freezing and sudden-transtion $d y$ namics of quantum discord under phase damping.

Theorem. The Bell-diagonal states of Eq. (2) undergoing the phase damping process can exhibit zero quantum discord rate, i.e., the so-called freezing phenomenon of discord, if and only if the parameters $\lambda_{i}$ 's satisfy

$$
\begin{aligned}
\lambda_{1} \lambda_{4} & =\lambda_{2} \lambda_{3}, \quad\left(\lambda_{1}-\lambda_{4}\right)\left(\lambda_{2}-\lambda_{3}\right)>0, \\
\text { or } \quad \lambda_{1} \lambda_{2} & =\lambda_{3} \lambda_{4},\left(\lambda_{1}-\lambda_{2}\right)\left(\lambda_{4}-\lambda_{3}\right)>0,
\end{aligned}
$$

alternatively. 
We specify that the inequalities in Eqs. (12a) and (12b) figure out different order relations for $\lambda_{i}$ 's. For instance, Eq. (12a) indicates the possible order as:

$$
\begin{array}{ll}
\lambda_{1}>\lambda_{2}>\lambda_{3}>\lambda_{4}, & \lambda_{4}>\lambda_{3}>\lambda_{2}>\lambda_{1}, \\
\lambda_{3}>\lambda_{4}>\lambda_{1}>\lambda_{2}, & \lambda_{2}>\lambda_{1}>\lambda_{4}>\lambda_{3} .
\end{array}
$$

To prove the theorem, let us suppose that one starts from a state of Eq. (2) in which the parameters $\lambda_{i}$ 's satisfy one of the order relations of Eq. (13). Note that any of these order relations yields that $c_{M}=\left|c_{1}\right|$, hence $Q_{A B}=Q_{A B}^{(1)}$, for the initial state. Consequently, the local variation of $Q_{A B}(q)$ during the starting time interval is illustrated by Eqs. (8)-(11). From the fact that the relative entropy $H_{2}(\mu \| \nu)$ is a strict convex function, the equality in between the first two expressions of Eq. (10) holds if and only if there are

$$
\mu(q)=\xi_{1}(q)=\xi_{2}(q), \quad \nu(q)=\eta_{1}(q)=\eta_{2}(q) .
$$

This leads clearly to the relation $\lambda_{1} \lambda_{4}=\lambda_{2} \lambda_{3}$, which is responsible for $\mathrm{d} Q_{A B}(0) / \mathrm{d} t=0$ of the specified initial state. It is crucial to note that according to Eq. (4), the relation $\lambda_{1}(q) \lambda_{4}(q)=\lambda_{2}(q) \lambda_{3}(q)$ will sustain during the evolution. The zero-rate dynamics of discord thus will continue until the presumed order relation is broken. In addition, it is direct to observe that a similar discussion is applicable for the condition of Eq. (12b) if one starts from a state with $c_{M}=\left|c_{2}\right|$. These facts together with that $\mathrm{d} Q_{A B}^{(3)}(q) / \mathrm{d} q \neq 0[\mathrm{cf}$. Eq. (6)] ] then complete our proof for the theorem. Straightforwardly, by substituting Eqs. (12a) or (12b) into Eq. (5) one obtains that

$$
Q_{A B}(q)=1-h_{2}\left(\lambda_{1}+\lambda_{3}\right)=Q_{A B}(0),
$$

where $h_{2}(x)$ stands for the aforementioned binary entropy function.

To shed light further on the physics related to the above derived condition of the freezing phenomenon, we notice that the term $H_{2}(\mu(q) \| \nu(q))$ in Eq. (8) can be rewritten as $H_{2}(\mu(q) \| \nu(q))=H_{4}\left(\xi^{\prime}(q) \| \eta^{\prime}(q)\right)$, where

$$
\begin{aligned}
\xi^{\prime}(q) & =\left\{\frac{x_{M}(q)}{2}, \frac{x_{M}(q)}{2}, \frac{1-x_{M}(q)}{2}, \frac{1-x_{M}(q)}{2}\right\}, \\
\eta^{\prime}(q) & =\left\{\frac{1-x_{M}(q)}{2}, \frac{1-x_{M}(q)}{2}, \frac{x_{M}(q)}{2}, \frac{x_{M}(q)}{2}\right\},
\end{aligned}
$$

and $x_{M}(q)=\lambda_{1}(q)+\lambda_{2}(q)$. Recall that the relative entropy $H_{n}(x \| y)$ tells actually how difficult it is to distinguish two states of the stochastic event, $\left\{x_{i}\right\}$ and $\left\{y_{i}\right\}$. The right hand of Eq. (8) can be understood as the reduction of the distinguishability of the two states undergoing a mixture process: $\xi(q) \rightarrow \xi^{\prime}(q)$ and $\eta(q) \rightarrow \eta^{\prime}(q)$, that is, the components of the first two of $\xi(q)$ and $\eta(q)$ are mixed and so well as the last two, respectively. At this stage, an alternative perspective on the freezing phenomenon of discord, respecting its necessary and sufficient conditions (12a) and (12b), is that the distinguishability of the two states $\xi(q)$ and $\eta(q)$ undergoing the specified stochastic mixture process will not alter as long as the corresponding condition is fulfilled.
With the necessary and sufficient conditions described above, we are now able to depict in Fig. 1 an overall geometric profile for those states in terms of parameters $\left\{\sqrt{\lambda_{1}}, \sqrt{\lambda_{2}}, \sqrt{\lambda_{3}}\right\}$. Regarding that $0 \leq \sum_{i=1}^{3} \lambda_{i} \leq 1$, the set of standard Bell-diagonal states constitute the portion of a unit sphere located in the first quadrant. The surface responsible for the conditions in Eqs. (12a) and (12b) comprises four leaf-shaped curves and the only common node of them corresponds to the maximally random state $\rho_{A B}=I / 4$. The boundary of the surface figures out the collection of transition points which can be described explicitly by four independent sets of equations as

$$
\begin{aligned}
& \lambda_{1}=\lambda_{2}=\sqrt{\lambda_{3}}-\lambda_{3}, \\
& \lambda_{3}=\lambda_{2}=\sqrt{\lambda_{1}}-\lambda_{1}, \\
& \lambda_{1}=1-\sqrt{\lambda_{2}}-\lambda_{3}=\left(1-\sqrt{\lambda_{2}}\right)^{2}, \\
& \lambda_{3}=1-\sqrt{\lambda_{2}}-\lambda_{1}=\left(1-\sqrt{\lambda_{2}}\right)^{2} .
\end{aligned}
$$

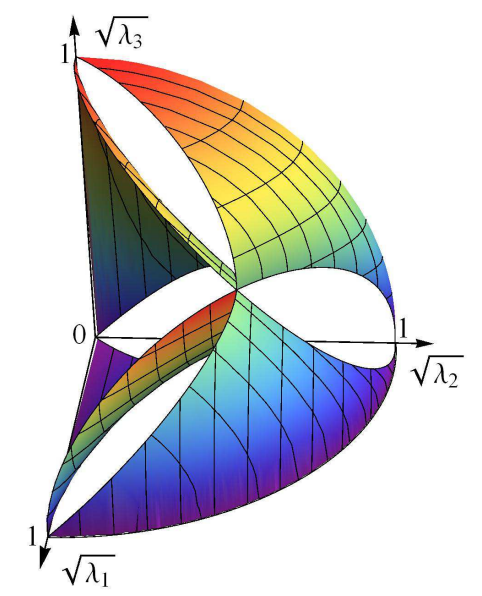

FIG. 1: Geometric profile in the parametric space of standard Bell-diagonal states that could exhibit freezing dynamics of quantum discord under phase damping.

The freezing and sudden transition phenomena of the dynamics are displayed schematically in Fig. 2. The trajectory of an initial state at the point $I$ proceeds along the surface with a constant discord till it encounters the boundary at the point $T$, after which the discord decreases to zero and the trajectory reaches finally the point $F$. At the transition point $q=q_{T}$, the rate of quantum discord suffers a sudden change from zero to a value of

$$
\left.\frac{\mathrm{d} Q_{A B}^{(3)}}{\mathrm{d} q}\right|_{q=q_{T}^{+}}=-\frac{1}{2\left(1-q_{T}\right)} H_{4}\left(\xi\left(q_{T}\right) \| \eta\left(q_{T}\right)\right),
$$

where we have used the relation of Eq. (6) and $\xi$ and $\eta$ are specified in Eq. (9).

It is worthwhile to point out that the conditions described in Eqs. (12a) and (12b) are applicable also to the frozen discord occurring in the non-Markovian dephasing process [12]. The dynamical evolution of this 
process can still be described phenomenologically by Eq. (11), hence the dependency of the discord on the parameter $q$ does not alter. As a result, all discussions related to Eqs. (12a) and (12b) are valid for its dynamics. Note that in the non-Markovian process $d(t)$ (hence $q$ ) is not a monotonic function of $t$ any more. Say, for the channel with random telegraph signal noise it is described as [22]

$$
q=1-d(t)=1-e^{-\gamma t}\left[\cos (\omega t)+\frac{\gamma}{\omega} \sin (\omega t)\right]
$$

where $\omega=\sqrt{4 a^{2}-\gamma^{2}}$, and $a$ characterizes the coupling strength of the system with environment. Differing from the model concerned in Ref. 12, in our system only one of the qubits is subjected to the noise channel. To display multiple transitions and freezing phenomena of the discord, the parameters of the initial state herein should satisfy $\left|c_{1(2)} / c_{3}\right|>e^{\pi \gamma / \omega}$. In the parametric space of $\left\{\sqrt{\lambda_{1}}, \sqrt{\lambda_{2}}, \sqrt{\lambda_{3}}\right\}$, we depict the evolution of the nonMarkovian process with $c_{1} / c_{3}=\frac{35}{24}$ and $\gamma / \omega=\frac{1}{32}$. It is shown that the trajectory manifests a traversing phenomenon between two separate leafs (see Fig. 2).

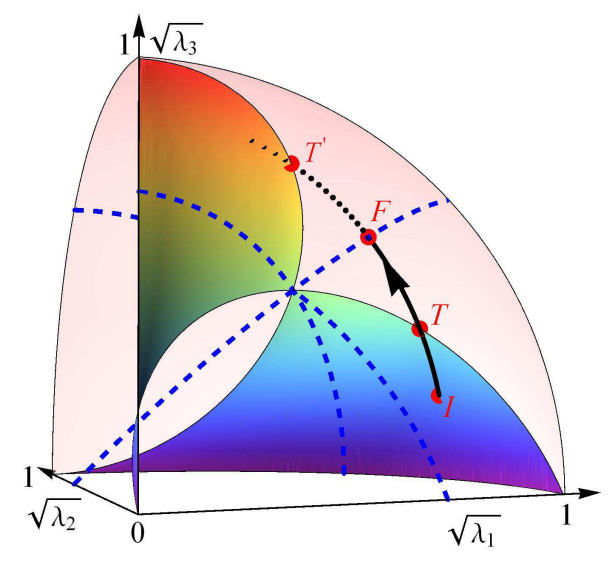

FIG. 2: Illustration of the trajectory for the freezing and sudden transition dynamics of quantum discord in the parametric space. The three blue-dashed lines account for the collection of Bell-diagonal states with zero discord and they share a common node with the two leaf-shaped curves determined by Eq. 12a . The initial state is specified as $\lambda_{1}=3 / 4, \lambda_{2}=3 / 16, \lambda_{3}=1 / 20$. The trajectory of the solid line proceeding along $I \rightarrow T \rightarrow F$ describes the evolution of the Markovian phase damping process. For a non-Markovian dephasing process specified by Eq. (19), the trajectory of the evolution proceeds further along the dotted line $F \rightarrow T^{\prime}$ and oscillates damply between the two specified leafs, displaying feedback and multiple transitions of the discord dynamics.
As a final proposal of this paper, we demonstrate that the above described freezing dynamics of discord and associated conditions will occur and coincide in a sort of new Bell-diagonal states beyond the standard form of Eq. (2). Consider that

$$
\rho_{A B}=\frac{1}{4}\left(I_{4}+\sum_{i=1}^{3} c_{i j} \sigma_{i}^{A} \otimes \sigma_{j}^{B}\right)=\sum_{i=1}^{4} \lambda_{i}^{\prime}\left|\psi_{i}^{\prime}\right\rangle\left\langle\psi_{i}^{\prime}\right|,
$$

where $c_{12} c_{21} \neq 0$ but all other off-diagonal elements of $\left\{c_{i j}\right\}$ vanish. The eigenstates are shown as $\left|\psi_{1,3}^{\prime}\right\rangle=$ $\frac{1}{\sqrt{2}}\left(|00\rangle \pm e^{-i \phi_{+}}|11\rangle\right),\left|\psi_{2,4}^{\prime}\right\rangle=\frac{1}{\sqrt{2}}\left(|01\rangle \pm e^{-i \phi_{-}}|10\rangle\right)$ with $\phi_{ \pm}=-\arctan \frac{c_{12} \pm c_{21}}{c_{22} \mp c_{11}}$, and the corresponding eigenvalues $\lambda_{i}^{\prime}$ 's relate to $c_{i j}$ 's via

$$
\begin{aligned}
& \lambda_{1,3}^{\prime}=\frac{1}{4}\left[1 \pm \sqrt{\left(c_{11}-c_{22}\right)^{2}+\left(c_{12}+c_{21}\right)^{2}}+c_{33}\right] \\
& \lambda_{2,4}^{\prime}=\frac{1}{4}\left[1 \pm \sqrt{\left(c_{11}+c_{22}\right)^{2}+\left(c_{12}-c_{21}\right)^{2}}-c_{33}\right] .
\end{aligned}
$$

Suppose that the subsystem $A$ is subjected to the local phase damping channel described by Eq. (1). It turns out that the state $\rho_{A B}(q)$ will keep the form of Eq. (20) with $\left\{c_{i j}\right\}$ evolving as: $c_{33}(q)=c_{33}$, and $c_{i j}(q)=(1-q) c_{i j}$ for all other elements. Consequently, the eigenvalues of $\rho_{A B}(q), \lambda_{i}^{\prime}(q)$, are derived promptly and they are verified to possess the similar expressions as those in Eq. (4). This implies that the discord of $\rho_{A B}(q)$ should display the same dynamics as states of Eq. (2). Therefore, it is safe to conclude that the freezing phenomenon could occur for the present state and the conditions of it are coincident with those in Eqs. (12a) and (12b).

In summary, we have studied freezing phenomena of quantum discord for Bell-diagonal states under phase damping noise processes. We have obtained necessary and sufficient conditions for these intriguing phenomena and the overall geometric profile is then illustrated in the parametric space for the corresponding states. Moreover, we have shown that the same freezing dynamics and conditions could occur and coincide in a sort of new Bell-diagonal states beyond the standard form of it. For further applications, our explicit derivation of the condition for frozen discord is of potential usefulness to realize quantum information processing, e.g., designing quantum systems to preserve at best the quantum coherence in the presence of the noisy environment.

This work was supported by the NSFC under grant No. 10874254.
[1] H. Ollivier and W. H. Zurek, Phys. Rev. Lett. 88, 017901 (2001).

[2] L. Henderson and V. Vedral, J. Phys. A 34, 6899 (2001).

[3] K. Modi, A. Brodutch, H. Cable, T. Paterek and V. Ve- dral, arXiv: 1112.6238, and references therein.

[4] A. Datta, A. Shaji, and C. M. Caves, Phys. Rev. Lett. 100, 050502 (2008).

[5] B. P. Lanyon, M. Barbieri, M. P. Almeida, and A. G. 
White, Phys. Rev. Lett. 101, 200501 (2008).

[6] T. Werlang, S. Souza, F. F. Fanchini, and C. J. Villas Boas, Phys. Rev. A 80, 024103 (2009).

[7] B. Wang, Z.-Y. Xu, Z.-Q. Chen, and M. Feng, Phys. Rev. A 81, 014101 (2010).

[8] J. Maziero, L. C. Céleri, R. M. Serra, and V. Vedral, Phys. Rev. A 80, 044102 (2009).

[9] J.-S. Xu, X.-Y. Xu, C.-F. Li, C.-J. Zhang, X.-B. Zou and G.-C. Guo, Nature Communications 1, 7 (2010).

[10] L. C. Céleri, A. G. S. Landulfo, R. M. Serra, and G. E. A. Matsas, Phys. Rev. A 81, 062130 (2010).

[11] L. Mazzola, J. Piilo, and S. Maniscalco, Phys. Rev. Lett. 104, 200401 (2010).

[12] L. Mazzola, J. Piilo, and S. Maniscalco, Int. Jour. Quan. Inf. 9, 981 (2011).

[13] R. Auccaise, L. C. Céleri, D. O. Soares-Pinto, et al., Phys. Rev. Lett. 107, 140403 (2011).

[14] B. Dakić, V. Vedral, Č. Brukner, Phys. Rev. Lett. 105, 190502 (2010).
[15] K. Modi, T. Paterek, W. Son, V. Vedral, and M. Williamson, Phys. Rev. Lett. 104, 080501 (2010).

[16] A. Streltsov, H. Kampermann, and D. Bruß, Phys. Rev. Lett. 107, 170502 (2011).

[17] Z. Y. Xu, W. L. Yang, X. Xiao, and M. Feng, J. Phys. A 44, 395304 (2011).

[18] B. Bellomo, R.L. Franco, and G. Compagno, arXiv: 1104.4043.

[19] S. Luo, Phys. Rev. A 77, 042303 (2008).

[20] Correspondingly, it is noted that during the time interval with $c_{M}(q)=\left|c_{i}(q)\right|$, the optimal projective measurement that achieves the maximization of the classical correlation $I_{c}\left(\rho_{A B}(q)\right)$ is given by $\Pi_{A}^{ \pm}(q)=\frac{1}{2}\left(I_{2} \pm \sigma_{i}\right)$.

[21] B. Schumacher and M. D. Westmoreland, eprint: quant-ph/0004045

[22] S. Daffer, K. Wódkiewicz, J.D. Cresser, and J.K. McIver, Phys. Rev. A 70, 010304(R) (2004). 